\title{
Review of: "Role of Ras in regulation of intestinal epithelial cell homeostasis and crosstalk with Wnt signaling"
}

Toshio Takahashi

Potential competing interests: The author(s) declared that no potential competing interests exist.

Kotani an coworkers revel that K-Ras is involved in regulation of intestinal epithelial cell (IEC) homeostasis, especially goblet and Paneth cells. Their previous study showed that ablation of Shp2 decreased the number of goblet cells and increased the Paneth cells (Yamashita et al. PLoS One 2014, 9(3):e92904). In the present research, the authors accomplish the molecular details of the new link between Shp2 and K-Ras signaling. Additionally, K-Ras activity has been shown to inhibit Wnt- $\beta$-catenin signaling using Wnt-target genes. Thus, the three-part mechanism of Shp2, K-Ras, and canonical Wnt signaling control IEC density in ileum and colon. I have a few concerns in the original article (PLoS One 2021, 16(8):e0256774). Lysozyme is a marker for Paneth cells in the small intestine (duodenum, jejunum, and ileum). In spite of common features, the epithelia of the small and colon show many differences. In particular, the colon lacks Paneth cells. Sasaki and coworkers showed that regenerating islet-derived protein 4 (Reg4) is a marker for deep crypt secretory cells (Sasaki et al. Proc. Natl. Acad. Sci. 2016, 113, E5399-E5407). It would be worth carrying out the expression pattern of Reg4 gene and/or Reg4-positive cells in the colon of IEC-RasDA mice. In Fig. 5, the authors show that K-Ras activity inhibits Wnt- $\beta$-catenin signaling. What kind of Wnts and their receptors, Frizzleds, are inhibited in wild-type mice? In contrast, what kind of Wnt molecules are enhanced in IEC-RasDA mice? When the questions are answered, we understand that K-Ras contributes to the proper regulation of intestinal epithelial cell homeostasis. 